\title{
NIELSEN THEORY, FLOER HOMOLOGY AND A GENERALISATION OF THE POINCARE - BIRKHOFF THEOREM
}

\author{
ALEXANDER FEL'SHTYN
}

\begin{abstract}
The purpose of this mostly expository paper is to discuss a connection between Nielsen fixed point theory and symplectic Floer homology for symplectomorphisms of surface and a calculation of Seidel's symplectic Floer homology for different mapping classes. We also describe symplectic zeta functions and an asymptotic symplectic invariant. A generalisation of the Poincare - Birkhoff fixed point theorem and Arnold conjecture is proposed.
\end{abstract}

\section{Contents}

1. Introduction

2. Nielsen fixed point theory and symplectic Floer homology

2.1. Symplectic Floer homology

2.2. Nielsen numbers and Floer homology

3. Symplectic zeta functions and asymptotic invariant

3.1. Symplectic zeta functions

3.2. Topological entropy and the Nielsen numbers

3.3. Asymptotic invariant

4. A generalisation of the Poincaré - Birkhoff theorem and Arnold conjecture. Concluding remarks

4.1. Algebraically finite mapping class

4.2. Pseudo-Anosov mapping class

4.3. Reducible mapping class

4.4. Concluding remarks

References

1991 Mathematics Subject Classification. 37C25; 53D; 37C30; 55M20.

Key words and phrases. Nielsen number, symplectic Floer homology, Poincare - Birkhoff theorem, Arnold conjecture . 


\section{InTRODUCTION}

Before discussing the main results of the paper, we briefly describe the few basic notions of Nielsen fixed point theory which will be used. We assume $X$ to be a connected, compact polyhedron and $f: X \rightarrow X$ to be a continuous map. Let $p: \tilde{X} \rightarrow X$ be the universal cover of $X$ and $\tilde{f}: \tilde{X} \rightarrow \tilde{X}$ a lifting of $f$, i.e. $p \circ \tilde{f}=f \circ p$. Two liftings $\tilde{f}$ and $\tilde{f}^{\prime}$ are called conjugate if there is a $\gamma \in \Gamma \cong \pi_{1}(X)$ such that $\tilde{f}^{\prime}=\gamma \circ \tilde{f} \circ \gamma^{-1}$. The subset $p(F i x(\tilde{f})) \subset F i x(f)$ is called the fixed point class of $f$ determined by the lifting class $[\tilde{f}]$. Two fixed points $x_{0}$ and $x_{1}$ of $f$ belong to the same fixed point class iff there is a path $c$ from $x_{0}$ to $x_{1}$ such that $c \cong f \circ c$ (homotopy relative endpoints). This fact can be considered as an equivalent definition of a non-empty fixed point class. Every map $f$ has only finitely many non-empty fixed point classes, each a compact subset of $X$. A fixed point class is called essential if its index is nonzero. The number of essential fixed point classes is called the Nielsen number of $f$, denoted by $N(f)$. The Nielsen number is always finite. $N(f)$ is a homotopy type invariant. In the category of compact, connected polyhedra, the Nielsen number of a map is, apart from certain exceptional cases, equal to the least number of fixed points of maps with the same homotopy type as $f$.

Recently a connection between symplectic Floer homology and Nielsen fixed point theory was discovered [14, 20]. The author came to the idea that Nielsen fixed point theory is connected with symplectic Floer homology theory of symplectomorphisms of surfaces at the Autumn 2000, after conversations with Joel Robbin and Dan Burghelea. This connection is based on the following simple fact: Gromov pseudoholomorphic curve in symplectic fixed point theory is nothing else as a Nielsen holomorphic disc. In the dimension two a diffeomorphism is symplectic if it preserves area. As a consequence, the symplectic geometry of surfaces lacks many of the interesting phenomena which are encountered in higher dimensions. For example, two symplectic automorphisms of a closed surface are symplectically isotopic iff they are homotopic, by a theorem of Moser [37]. On other hand symplectic fixed point theory is very nontrivial in dimension 2. A selebrated landmark in this subject is Poincaré's last geometric theorem, proved by Birkhoff [1] in the 1925, which asserts that an area-preserving twist map of the annulus must have at least two distinct 
fixed points. It is known that symplectic Floer homology for surface symplectomorphism is the $d=1$ part of periodic Floer homology of this symplectomorphism [24], a Floer homology theory whose chain complex is generated by certain multisets of periodic orbits and whose differentials count certain embedded pseudoholomorphic curves. This theory is conjectured [24] to be isomorphic to the Seiberg - Witten Floer homology [33] of the mapping torus of symplectomorphism in certain spin ${ }^{c_{-}}$ structures and also is conjectured to agree with Heegaard Floer homology $\mathrm{HF}^{+}[38$ of the mapping torus. It is known also that symplectic Floer homology of symplectomorphism of surface is a simple model for the instanton Floer homology of the mapping torus of this symplectomorphism [6].

In the chapter 2 we discuss the connection between symplectic Floer homology theory and Nielsen fixed point theory and the calculations of Seidel's symplectic Floer homology for periodic [20, 14] and algebraically finite mapping classes [20]. In the chapter 3 we describe symplectic zeta functions and an asymptotic invariant of monotone symplectomorphism. In the chapter 4 we discuss a generalisation of the Poincaré - Birkhoff theorem and Arnold conjecture and the calculations of Seidel's symplectic Floer homology for pseudo-Anosov and reducible mapping classes [3] The results of this paper were announced on the Symplectic Geometry Seminars at Stanford and Princeton in April and October, 2007.

The author is grateful to A. Cotton-Clay, Ya. Eliashberg, Wu-Chung Hsiang, E. Ionel, J. Kedra, K. Ono, Y. Rudyak, G. Tian, V. Turaev and M. Usher for stimulating discussions and comments.

Parts of this article were written while the author was visiting the Max-PlanckInstitute für Mathematik, Bonn in 2004-2005. The author would like to thank the Max-Planck-Institute für Mathematik, Bonn for kind hospitality and support.

\section{Nielsen fiXed POINT theORY AND SympleCtiC Floer homology}

\subsection{Symplectic Floer homology.}

2.1.1. Monotonicity. In this section we discuss the notion of monotonicity as defined in [41, 20]. Monotonicity plays important role for Floer homology in two dimensions. Throughout this article, $M$ denotes a closed connected and oriented 2-manifold of genus $\geq 2$. Pick an everywhere positive two-form $\omega$ on $M$. 
Let $\phi \in \operatorname{Symp}(M, \omega)$, the group of symplectic automorphisms of the two-dimensional symplectic manifold $(M, \omega)$. The mapping torus of $\phi, T_{\phi}=\mathbb{R} \times M /(t+1, x) \sim$ $(t, \phi(x))$, is a 3 -manifold fibered over $S^{1}=\mathbb{R} / \mathbb{Z}$. There are two natural second cohomology classes on $T_{\phi}$, denoted by $\left[\omega_{\phi}\right]$ and $c_{\phi}$. The first one is represented by the closed two-form $\omega_{\phi}$ which is induced from the pullback of $\omega$ to $\mathbb{R} \times M$. The second is the Euler class of the vector bundle $V_{\phi}=\mathbb{R} \times T M /\left(t+1, \xi_{x}\right) \sim\left(t, \mathrm{~d} \phi_{x} \xi_{x}\right)$, which is of rank 2 and inherits an orientation from $T M$.

$\phi \in \operatorname{Symp}(M, \omega)$ is called monotone, if $\left[\omega_{\phi}\right]=\left(\operatorname{area}_{\omega}(M) / \chi(M)\right) \cdot c_{\phi}$ in $H^{2}\left(T_{\phi} ; \mathbb{R}\right) ;$ throughout this article $\operatorname{Symp}^{m}(M, \omega)$ denotes the set of monotone symplectomorphisms.

Now $H^{2}\left(T_{\phi} ; \mathbb{R}\right)$ fits into the following short exact sequence [41, 20]

$$
0 \longrightarrow \frac{H^{1}(M ; \mathbb{R})}{\text { image }\left(\mathrm{id}-\phi^{*}\right)} \stackrel{d}{\longrightarrow} H^{2}\left(T_{\phi} ; \mathbb{R}\right) \stackrel{r^{*}}{\longrightarrow} H^{2}(M ; \mathbb{R}), \longrightarrow 0 .
$$

where the map $r^{*}$ is restriction to the fiber. The map $d$ is defined as follows. Let $\rho: I \rightarrow \mathbb{R}$ be a smooth function which vanishes near 0 and 1 and satisfies $\int_{0}^{1} \rho \mathrm{d} t=1$. If $\theta$ is a closed 1 -form on $M$, then $\rho \cdot \theta \wedge \mathrm{d} t$ defines a closed 2-form on $T_{\phi}$; indeed $d[\theta]=[\rho \cdot \theta \wedge \mathrm{d} t]$. The map $r: M \hookrightarrow T_{\phi}$ assigns to each $x \in M$ the equivalence class of $(1 / 2, x)$. Note, that $r^{*} \omega_{\phi}=\omega$ and $r^{*} c_{\phi}$ is the Euler class of $T M$. Hence, by (2.1), there exists a unique class $m(\phi) \in H^{1}(M ; \mathbb{R}) /$ image $\left(\mathrm{id}-\phi^{*}\right)$ satisfying $d m(\phi)=\left[\omega_{\phi}\right]-\left(\operatorname{area}_{\omega}(M) / \chi(M)\right) \cdot c_{\phi}$, where $\chi(M)$ denotes the Euler characteristic of $M$. Therefore, $\phi$ is monotone if and only if $m(\phi)=0$.

Because $c_{\phi}$ controls the index, or expected dimension, of moduli spaces of holomorphic curves under change of homology class and $\omega_{\phi}$ controls their energy under change of homology class, the monotonicity condition ensures that the energy is constant on the index one components of the moduli space, which implies compactness and, as a corollary, finite count in a differential of the Floer complex.

We recall the fundamental properties of $\operatorname{Symp}^{m}(M, \omega)$ from [41, 20]. Let $\operatorname{Diff}^{+}(M)$ denotes the group of orientation preserving diffeomorphisms of $M$.

(Identity) $\operatorname{id}_{M} \in \operatorname{Symp}^{m}(M, \omega)$.

(Naturality) If $\phi \in \operatorname{Symp}^{m}(M, \omega), \psi \in \operatorname{Diff}^{+}(M)$, then $\psi^{-1} \phi \psi \in \operatorname{Symp}^{m}\left(M, \psi^{*} \omega\right)$.

(Isotopy) Let $\left(\psi_{t}\right)_{t \in I}$ be an isotopy $\operatorname{in} \operatorname{Symp}(M, \omega)$, i.e. a smooth path with $\psi_{0}=$ id. Then $m\left(\phi \circ \psi_{1}\right)=m(\phi)+\left[\operatorname{Flux}\left(\psi_{t}\right)_{t \in I}\right]$ in $H^{1}(M ; \mathbb{R}) /$ image $\left(\mathrm{id}-\phi^{*}\right)$; see [41, Lemma 
6]. For the definition of the flux homomorphism see [35].

(Inclusion) The inclusion $\operatorname{Symp}^{m}(M, \omega) \hookrightarrow \operatorname{Diff}^{+}(M)$ is a homotopy equivalence.

(Floer homology) To every $\phi \in \operatorname{Symp}^{m}(M, \omega)$ symplectic Floer homology theory assigns a $\mathbb{Z}_{2}$-graded vector space $H F_{*}(\phi)$ over $\mathbb{Z}_{2}$, with an additional multiplicative structure, called the quantum cap product, $H^{*}\left(M ; \mathbb{Z}_{2}\right) \otimes H F_{*}(\phi) \longrightarrow H F_{*}(\phi)$. For $\phi=\mathrm{id}_{M}$ the symplectic Floer homology $H F_{*}\left(\mathrm{id}_{M}\right)$ are canonically isomorphic to ordinary homology $H_{*}\left(M ; \mathbb{Z}_{2}\right)$ and quantum cap product agrees with the ordinary cap product. Each $\psi \in \operatorname{Diff}^{+}(M)$ induces an isomorphism $H F_{*}(\phi) \cong H F_{*}\left(\psi^{-1} \phi \psi\right)$ of $H^{*}\left(M ; \mathbb{Z}_{2}\right)$-modules.

(Invariance) If $\phi, \phi^{\prime} \in \operatorname{Symp}^{m}(M, \omega)$ are isotopic, then $H F_{*}(\phi)$ and $H F_{*}\left(\phi^{\prime}\right)$ are naturally isomorphic as $H^{*}\left(M ; \mathbb{Z}_{2}\right)$-modules. This is proven in [41, Page 7]. Note that every Hamiltonian perturbation of $\phi$ (see [6]) is also in $\operatorname{Symp}^{m}(M, \omega)$.

Now let $g$ be a mapping class of $M$, i.e. an isotopy class of $\operatorname{Diff}^{+}(M)$. Pick an area form $\omega$ and a representative $\phi \in \operatorname{Symp}^{m}(M, \omega)$ of $g$. Then $H F_{*}(\phi)$ is an invariant of $g$, which is denoted by $H F_{*}(g)$. Note that $H F_{*}(g)$ is independent of the choice of an area form $\omega$ by Moser's isotopy theorem [37] and naturality of Floer homology.

2.1.2. Floer homology. Let $\phi \in \operatorname{Symp}(M, \omega)$. There are two ways of constructing Floer homology detecting its fixed points, $\operatorname{Fix}(\phi)$. Firstly, the graph of $\phi$ is a Lagrangian submanifold of $M \times M,(-\omega) \times \omega)$ and its fixed points correspond to the intersection points of graph $(\phi)$ with the diagonal $\Delta=\{(x, x) \in M \times M\}$. Thus we have the Floer homology of the Lagrangian intersection $H F_{*}(M \times M, \Delta, \operatorname{graph}(\phi))$. This intersection is transversal if the fixed points of $\phi$ are nondegenerate, i.e. if 1 is not an eigenvalue of $d \phi(x)$, for $x \in \operatorname{Fix}(\phi)$. The second approach was mentioned by Floer in [17] and presented with details by Dostoglou and Salamon in [6. We follow here Seidel's approach [41] which, comparable with [6], uses a larger class of perturbations, but such that the perturbed action form is still cohomologous to the unperturbed. As a consequence, the usual invariance of Floer homology under Hamiltonian isotopies is extended to the stronger property stated above. Let now $\phi \in \operatorname{Symp}^{m}(M, \omega)$, i.e $\phi$ is monotone. Firstly, we give the definition of $H F_{*}(\phi)$ in the special case where all the fixed points of $\phi$ are non-degenerate, i.e. for all $y \in \operatorname{Fix}(\phi)$, $\operatorname{det}\left(\mathrm{id}-\mathrm{d} \phi_{y}\right) \neq 0$, and then following Seidels approach [41] we consider general case when $\phi$ has degenerate fixed points. Let $\Omega_{\phi}=\left\{y \in C^{\infty}(\mathbb{R}, M) \mid y(t)=\phi(y(t+1))\right\}$ 
be the twisted free loop space, which is also the space of sections of $T_{\phi} \rightarrow S^{1}$. The action form is the closed one-form $\alpha_{\phi}$ on $\Omega_{\phi}$ defined by

$$
\alpha_{\phi}(y) Y=\int_{0}^{1} \omega(d y / d t, Y(t)) d t .
$$

where $y \in \Omega_{\phi}$ and $Y \in T_{y} \Omega_{\phi}$, i.e. $Y(t) \in T_{y(t)} M$ and $Y(t)=\mathrm{d} \phi_{y(t+1)} Y(t+1)$ for all $t \in \mathbb{R}$.

The tangent bundle of any symplectic manifold admits an almost complex structure $J: T M \longrightarrow T M$ which is compatible with $\omega$ in sense that $(v, w)=\omega(v, J w)$ defines a Riemannian metric. Let $J=\left(J_{t}\right)_{t \in \mathbb{R}}$ be a smooth path of $\omega$-compatible almost complex structures on $M$ such that $J_{t+1}=\phi^{*} J_{t}$. If $Y, Y^{\prime} \in T_{y} \Omega_{\phi}$, then $\int_{0}^{1} \omega\left(Y^{\prime}(t), J_{t} Y(t)\right) \mathrm{d} t$ defines a metric on the loop space $\Omega_{\phi}$. So the critical points of $\alpha_{\omega}$ are the constant paths in $\Omega_{\phi}$ and hence the fixed points of $\phi$. The negative gradient lines of $\alpha_{\omega}$ with respect to the metric above are solutions of the partial differential equations with boundary conditions

$$
\left\{\begin{array}{l}
u(s, t)=\phi(u(s, t+1)) \\
\partial_{s} u+J_{t}(u) \partial_{t} u=0 \\
\lim _{s \rightarrow \pm \infty} u(s, t) \in \operatorname{Fix}(\phi)
\end{array}\right.
$$

These are exactly Gromov's pseudoholomorphic curves [21].

For $y^{ \pm} \in \operatorname{Fix}(\phi)$, let $\mathcal{M}\left(y^{-}, y^{+} ; J, \phi\right)$ denote the space of smooth maps $u: \mathbb{R}^{2} \rightarrow M$ which satisfy the equations (2.2). Now to every $u \in \mathcal{M}\left(y^{-}, y^{+} ; J, \phi\right)$ we associate a Fredholm operator $\mathrm{D}_{u}$ which linearizes (2.2) in suitable Sobolev spaces. The index of this operator is given by the so called Maslov index $\mu(u)$, which satisfies $\mu(u)=\operatorname{deg}\left(y^{+}\right)-\operatorname{deg}\left(y^{-}\right) \bmod 2$, where $(-1)^{\operatorname{deg} y}=\operatorname{sign}\left(\operatorname{det}\left(\mathrm{id}-\mathrm{d} \phi_{y}\right)\right)$. We have no bubbling, since for surface $\pi_{2}(M)=0$. For a generic $J$, every $u \in \mathcal{M}\left(y^{-}, y^{+} ; J, \phi\right)$ is regular, meaning that $\mathrm{D}_{u}$ is onto. Hence, by the implicit function theorem, $\mathcal{M}_{k}\left(y^{-}, y^{+} ; J, \phi\right)$ is a smooth $k$-dimensional manifold, where $\mathcal{M}_{k}\left(y^{-}, y^{+} ; J, \phi\right)$ denotes the subset of those $u \in \mathcal{M}\left(y^{-}, y^{+} ; J, \phi\right)$ with $\mu(u)=k \in \mathbb{Z}$. Translation of the $s$-variable defines a free $\mathbb{R}$-action on 1 -dimensional manifold $\mathcal{M}_{1}\left(y^{-}, y^{+} ; J, \phi\right)$ and hence the quotient is a discrete set of points. The energy of a map $u: \mathbb{R}^{2} \rightarrow M$ is given by $E(u)=\int_{\mathbb{R}} \int_{0}^{1} \omega\left(\partial_{t} u(s, t), J_{t} \partial_{t} u(s, t)\right) \mathrm{d} t \mathrm{~d} s$ for all $y \in \operatorname{Fix}(\phi)$. P.Seidel has proved in [41] that if $\phi$ is monotone, then the energy is constant on each $\mathcal{M}_{k}\left(y^{-}, y^{+} ; J, \phi\right)$. Since all fixed points of $\phi$ are nondegenerate the set $\operatorname{Fix}(\phi)$ is 
a finite set and the $\mathbb{Z}_{2}$-vector space $C F_{*}(\phi):=\mathbb{Z}_{2}^{\# \operatorname{Fix}(\phi)}$ admits a $\mathbb{Z}_{2}$-grading with $(-1)^{\operatorname{deg} y}=\operatorname{sign}\left(\operatorname{det}\left(\mathrm{id}-\mathrm{d} \phi_{y}\right)\right)$, for all $y \in \operatorname{Fix}(\phi)$. The boundedness of the energy $E(u)$ for monotone $\phi$ implies that the 0 -dimensional quotients $\mathcal{M}_{1}\left(y_{-}, y_{+}, J, \phi\right) / \mathbb{R}$ are actually finite sets. Denoting by $n\left(y_{-}, y_{+}\right)$the number of points mod 2 in each of them, one defines a differential $\partial_{J}: C F_{*}(\phi) \rightarrow C F_{*+1}(\phi)$ by $\partial_{J} y_{-}=$ $\sum_{y_{+}} n\left(y_{-}, y_{+}\right) y_{+}$. Due to gluing theorem this Floer boundary operator satisfies $\partial_{J} \circ \partial_{J}=0$. For gluing theorem to hold one needs again the boundedness of the energy $E(u)$. It follows that $\left(C F_{*}(\phi), \partial_{J}\right)$ is a chain complex and its homology is by definition the Floer homology of $\phi$ denoted $H F_{*}(\phi)$. It is independent of $J$ and is an invariant of $\phi$.

If $\phi$ has degenerate fixed points one needs to perturb equations (2.2) in order to define the Floer homology. Equivalently, one could say that the action form needs to be perturbed. The necessary analysis is given in [41], it is essentially the same as in the slightly different situations considered in [6]. But Seidel's approach also differs from the usual one in [6]. He uses a larger class of perturbations, but such that the perturbed action form is still cohomologous to the unperturbed.

\subsection{Nielsen numbers and Floer homology.}

\subsubsection{Periodic diffeomorphisms.}

Lemma 2.1. 29] Let $\phi$ a non-trivial orientation preserving periodic diffeomorphism of a compact connected surface $M$ of Euler characteristic $\chi(M) \leq 0$. Then each fixed point class of $\phi$ consists of a single point which has index 1.

There are two criteria for monotonicity which we use later on. Let $\omega$ be an area form on $M$ and $\phi \in \operatorname{Symp}(M, \omega)$.

Lemma 2.2. [20] Assume that every class $\alpha \in \operatorname{ker}\left(\mathrm{id}-\phi_{*}\right) \subset H_{1}(M ; \mathbb{Z})$ is represented by a map $\gamma: S \rightarrow \operatorname{Fix}(\phi)$, where $S$ is a compact oriented 1-manifold. Then $\phi$ is monotone.

Lemma 2.3. [20] If $\phi^{k}$ is monotone for some $k>0$, then $\phi$ is monotone. If $\phi$ is monotone, then $\phi^{k}$ is monotone for all $k>0$.

We shall say that $\phi: M \rightarrow M$ is a periodic map of period $m$, if $\phi^{m}$ is the identity $\operatorname{map~id}_{M}: M \rightarrow M$. 
Theorem 2.4. [14] If $\phi$ is a non-trivial orientation preserving periodic diffeomorphism of a compact connected surface $M$ of Euler characteristic $\chi(M) \leq 0$, then $\phi$ is monotone symplectomorphism with respect to some $\phi$-invariant area form and

$$
H F_{*}(\phi) \cong \mathbb{Z}_{2}^{N(\phi)}
$$

where $N(\phi)$ denotes the Nielsen number of $\phi$.

Proof. Let $\phi$ be a periodic diffeomorphism of least period $l$. First note that if $\tilde{\omega}$ is an area form on $M$, then area form $\omega:=\sum_{i=1}^{\ell}\left(\phi^{i}\right)^{*} \tilde{\omega}$ is $\phi$-invariant, i.e. $\phi \in$

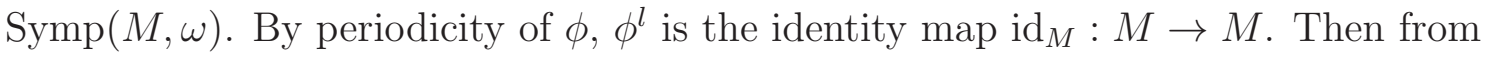
Lemmas 2.2 and 2.3 it follows that $\omega$ can be chosen such that $\phi \in \operatorname{Symp}^{m}(M, \omega)$.

Lemma 2.1 implies that every $y \in \operatorname{Fix}(\phi)$ forms a different fixed point class of $\phi$, so $\# \operatorname{Fix}(\phi)=N(\phi)$. This has an immediate consequence for the Floer complex $\left(C F_{*}(\phi), \partial_{J}\right)$ with respect to a generic $J=\left(J_{t}\right)_{t \in \mathbb{R}}$. If $y^{ \pm} \in \operatorname{Fix}(\phi)$ are in different fixed point classes, then $\mathcal{M}\left(y^{-}, y^{+} ; J, \phi\right)=\emptyset$. This follows from the first equation in (2.2). Then the boundary map in the Floer complex is zero $\partial_{J}=0$ and $\mathbb{Z}_{2}$-vector space $C F_{*}(\phi):=\mathbb{Z}_{2}^{\# \operatorname{Fix}(\phi)}=\mathbb{Z}_{2}^{N(\phi)}$. This immediately implies $H F_{*}(\phi) \cong \mathbb{Z}_{2}^{N(\phi)}$ and $\operatorname{dim} H F_{*}(\phi)=N(\phi)$.

2.2.2. Algebraically finite mapping classes. A mapping class of $M$ is called algebraically finite if it does not have any pseudo-Anosov components in the sense of Thurston's theory of surface diffeomorphism. The term algebraically finite goes back to J. Nielsen [39].

In [20] the diffeomorphisms of finite type were defined. These are special representatives of algebraically finite mapping classes adopted to the symplectic geometry.

Definition 2.1. [20] We call $\phi \in \operatorname{Diff}_{+}(M)$ of finite type if the following holds. There is a $\phi$-invariant finite union $N \subset M$ of disjoint non-contractible annuli such that:

(1) $\phi \mid M \backslash N$ is periodic, i.e. there exists $\ell>0$ such that $\phi^{\ell} \mid M \backslash N=$ id.

(2) Let $N^{\prime}$ be a connected component of $N$ and $\ell^{\prime}>0$ be the smallest integer such that $\phi^{\ell^{\prime}}$ maps $N^{\prime}$ to itself. Then $\phi^{\ell^{\prime}} \mid N^{\prime}$ is given by one of the following two models with respect to some coordinates $(q, p) \in I \times S^{1}$ : 
(twist map)

$$
(q, p) \longmapsto(q, p-f(q))
$$

(flip-twist map)

$$
(q, p) \longmapsto(1-q,-p-f(q)),
$$

where $f: I \rightarrow \mathbb{R}$ is smooth and strictly monotone. A twist map is called positive or negative, if $f$ is increasing or decreasing.

(3) Let $N^{\prime}$ and $\ell^{\prime}$ be as in (2). If $\ell^{\prime}=1$ and $\phi \mid N^{\prime}$ is a twist map, then image $(f) \subset$ $[0,1]$, i.e. $\phi \mid \operatorname{int}\left(N^{\prime}\right)$ has no fixed points.

(4) If two connected components of $N$ are homotopic, then the corresponding local models of $\phi$ are either both positive or both negative twists.

The term flip-twist map is taken from [31].

By $M_{\text {id }}$ we denote the union of the components of $M \backslash \operatorname{int}(N)$, where $\phi$ restricts to the identity.

The next lemma describes the set of fixed point classes of $\phi$. It is a special case of a theorem by B. Jiang and J. Guo [31], which gives for any mapping class a representative that realizes its Nielsen number.

Lemma 2.5 (Fixed point classes). [31] Each fixed point class of $\phi$ is either a connected component of $M_{\mathrm{id}}$ or consists of a single fixed point. A fixed point $x$ of the second type satisfies $\operatorname{det}\left(\mathrm{id}-\mathrm{d} \phi_{x}\right)>0$.

The monotonicity of diffeomorphisms of finite type was investigated in details in [20]. Let $\phi$ be a diffeomorphism of finite type and $\ell$ be as in (1). Then $\phi^{\ell}$ is the product of (multiple) Dehn twists along $N$. Moreover, two parallel Dehn twists have the same sign, by (4). We say that $\phi$ has uniform twists, if $\phi^{\ell}$ is the product of only positive, or only negative Dehn twists.

Furthermore, we denote by $\ell$ the smallest positive integer such that $\phi^{\ell}$ restricts to the identity on $M \backslash N$.

If $\omega^{\prime}$ is an area form on $M$ which is the standard form $\mathrm{d} q \wedge \mathrm{d} p$ with respect to the $(q, p)$-coordinates on $N$, then $\omega:=\sum_{i=1}^{\ell}\left(\phi^{i}\right)^{*} \omega^{\prime}$ is standard on $N$ and $\phi$-invariant, i.e. $\phi \in \operatorname{Symp}(M, \omega)$. To prove that $\omega$ can be chosen such that $\phi \in \operatorname{Symp}^{m}(M, \omega)$, Gautschi distinguishes two cases: uniform and non-uniform twists. In the first case he proves the following stronger statement. 
Lemma 2.6. [20] If $\phi$ has uniform twists and $\omega$ is a $\phi$-invariant area form, then $\phi \in \operatorname{Symp}^{m}(M, \omega)$.

In the non-uniform case, monotonicity does not hold for arbitrary $\phi$-invariant area forms.

Lemma 2.7. [20] If $\phi$ does not have uniform twists, there exists a $\phi$-invariant area form $\omega$ such that $\phi \in \operatorname{Symp}^{m}(M, \omega)$. Moreover, $\omega$ can be chosen such that it is the standard form $\mathrm{d} q \wedge \mathrm{d} p$ on $N$.

Theorem 2.8. [14] If $\phi$ is a diffeomorphism of finite type of a compact connected surface $M$ of Euler characteristic $\chi(M)<0$ and if $\phi$ has only isolated fixed points, then $\phi$ is monotone with respect to some $\phi$-invariant area form and

$$
H F_{*}(\phi) \cong \mathbb{Z}_{2}^{N(\phi)}
$$

where $N(\phi)$ denotes the Nielsen number of $\phi$.

Proof. From Lemmas 2.6 and 2.7 it follows that $\omega$ can be chosen such that $\phi \in$ $\operatorname{Symp}^{m}(M, \omega)$. Lemma 2.5 implies that every $y \in \operatorname{Fix}(\phi)$ forms a different fixed point class of $\phi$, so \# $\operatorname{Fix}(\phi)=N(\phi)$. This has an immediate consequence for the Floer complex $\left(C F_{*}(\phi), \partial_{J}\right)$ with respect to a generic $J=\left(J_{t}\right)_{t \in \mathbb{R}}$. If $y^{ \pm} \in \operatorname{Fix}(\phi)$ are in different fixed point classes, then $\mathcal{M}\left(y^{-}, y^{+} ; J, \phi\right)=\emptyset$. This follows from the first equation in (2.2). Then the boundary map in the Floer complex is zero $\partial_{J}=0$ and $\mathbb{Z}_{2}$-vector space $C F_{*}(\phi):=\mathbb{Z}_{2}^{\# \operatorname{Fix}(\phi)}=\mathbb{Z}_{2}^{N(\phi)}$. This immediately implies $H F_{*}(\phi) \cong \mathbb{Z}_{2}^{N(\phi)}$.

Theorem 2.9. [20] Let $\phi$ be a diffeomorphism of finite type, then $\phi$ is monotone with respect to some $\phi$-invariant area form and

$$
H F_{*}(\phi)=H_{*}\left(M_{\mathrm{id}}, \partial_{M_{\mathrm{id}}} ; \mathbb{Z}_{2}\right) \oplus \mathbb{Z}_{2}^{L\left(\phi \mid M \backslash M_{\mathrm{id}}\right)} .
$$

Here, L denotes the Lefschetz number.

Proof. The main idea of the proof is a separation mechanism for Floer connecting orbits. Together with the topological separation of fixed points discussed in theorem 2.8, it allows us to compute the Floer homology of diffeomorphisms of finite type. 
There exists a function $H: M \rightarrow \mathbb{R}$ such that $H \mid \operatorname{int}\left(M_{i d}\right)$ is a Morse function, meaning that all the critical points are non-degenerate and $H \mid\left(M \backslash M_{i d}\right)=0$. Let $\left(\psi_{t}\right)_{t \in \mathbb{R}}$ denote the Hamiltonian flow generated by $H$ with respect to the fixed area form $\omega$ and set $\Phi:=\phi \circ \psi_{1}$. Then Fix $\Phi=\left(\operatorname{crit}(H) \cap M_{i d}\right) \cup\left(\operatorname{Fix} \phi \backslash M_{i d}\right)$. In particular, $\Phi$ only has non-degenerate fixed points. Let $N_{0} \subset M_{i d}$ be a collar neighborhood of $\partial M_{i d}$. Let $x^{-}, x^{+} \in \operatorname{Fix} \Phi \cap M_{i d}$ be in the same connected component of $M_{i d}$. If $u \in \mathcal{M}\left(x^{-}, x^{+} ; J, \Phi\right)$, then image $u \subset M_{\delta}$, where $M_{\delta}$ denotes the $\delta$-neighborhood of $M_{i d} \backslash N_{0}$ with respect to any of the metrics $\omega\left(., J_{t}.\right)$ [41, 20] Moreover, lemma 2.5 implies that every $y \in \operatorname{Fix}(\phi) \backslash M_{i d}$ forms a different fixed point class of $\Phi$. This has an immediate consequence for the Floer complex $\left(C F_{*}(\Phi), \partial_{J}\right)$ with respect to a generic $J=\left(J_{t}\right)_{t \in \mathbb{R}}$. Namely, $\left(C F_{*}(\Phi), \partial_{J}\right)$ splits into the subcomplexes $\left(\mathcal{C}_{1}, \partial_{1}\right)$ and $\left(\mathcal{C}_{2}, \partial_{2}\right)$, where $\mathcal{C}_{1}$ is generated by $\operatorname{crit}(H) \cap M_{i d}$ and $\mathcal{C}_{2}$ by $\operatorname{Fix}(\phi) \backslash M_{i d}$. Moreover, $\mathcal{C}_{2}$ is graded by 0 and $\partial_{2}=0$ [20]. The homology of $\left(\mathcal{C}_{1}, \partial_{1}\right)$ is isomorphic

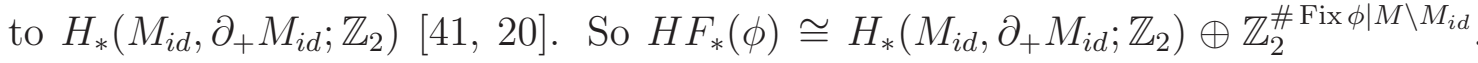
Since every fixed point of $\phi \mid M \backslash M_{i d}$ has fixed point index 1, the Lefschetz fixed point formula implies that $\#\left(\operatorname{Fix} \phi \backslash M_{i d}\right)=L\left(\phi \mid\left(M \backslash M_{i d}\right)\right)$.

Remark 2.2. In the theorem 2.8 the set $M_{\text {id }}$ is empty and every fixed point of $\phi$ has fixed point index 1 [31. The Lefschetz fixed point formula implies that $\#$ Fix $\phi=N(\phi)=L(\phi)$. So, theorem 2.8 follows also from theorem 2.9.

2.2.3. Anosov diffeomorphisms of 2-dimensional torus. The connection between Nielsen number and the dimension of symplectic Floer homology remains true for genus 1 surface and Anosov diffeomorphism .

Theorem 2.10. [14] If $\phi$ is a Anosov diffeomorphism of a 2-dimensional torus $T^{2}$, then $\phi$ is symplectic and

$$
H F_{*}(\phi) \cong \mathbb{Z}_{2}^{N(\phi)}
$$

where $N(\phi)=\left|\operatorname{det}\left(E-\phi_{*}\right)\right|$ denotes the Nielsen number of $\phi$ and $\phi_{*}$ is an induced homomorphism on the fundamental group of $T^{2}$.

Proof. Hyperbolicity of $\phi$ means that the covering linear map $\tilde{\phi}: R^{2} \rightarrow R^{2}, \operatorname{det} \tilde{\phi}=1$ has no eigenvalue of modulus one. The Anosov diffeomorphism of a 2-dimensional torus $T^{2}$ is area preserving so symplectic. In fact, the covering map $\tilde{\phi}$ has a unique 
fixed point, which is the origin; hence, by the covering homotopy theorem, the fixed points of $\phi$ are pairwise Nielsen nonequivalent.The index of each Nielsen fixed point class, consisting of one fixed point, coincides with its Lefschetz index, and by the hyperbolicity of $\phi$, the later is not equal to zero.Thus the Nielsen number $N(\phi)=\# \operatorname{Fix}(\phi)$. If $y^{ \pm} \in \operatorname{Fix}(\phi)$ are in different Nielsen fixed point classes, then $\mathcal{M}\left(y^{-}, y^{+} ; J, \phi\right)=\emptyset$. This follows from the first equation in (2.2). Monotonicity condition trivially follows from the isomorphism $H_{2}(M, \mathbb{R}) \cong H_{2}\left(T_{\phi}, \mathbb{R}\right)$. Then the boundary map in the Floer complex is zero $\partial_{J}=0$ and $\mathbb{Z}_{2}$-vector space $C F_{*}(\phi):=$ $\mathbb{Z}_{2}^{\# \operatorname{Fix}(\phi)}=\mathbb{Z}_{2}^{N(\phi)}$

This immediately implies $H F_{*}(\phi) \cong \mathbb{Z}_{2}^{N(\phi)}$.

\section{SyMPLECTIC ZETA FUNCTIONS AND ASYMPTOTIC INVARIANT}

3.1. Symplectic zeta functions. Let $\operatorname{Mod}_{M}=\pi_{0}\left(\operatorname{Diff} f^{+}(M)\right)$ be the mapping class group of a closed connected oriented surface $M$ of genus $\geq 2$. Pick an everywhere positive two-form $\omega$ on $M$. A isotopy theorem of Moser [37] says that each mapping class of $g \in \Gamma$, i.e. an isotopy class of $D i f f^{+}(M)$, admits representatives which preserve $\omega$. Due to Seidel[41] we can pick a monotone representative $\phi \in \operatorname{Symp}^{m}(M, \omega)$ of $g$. Then $H F_{*}(\phi)$ is an invariant of $g$, which is denoted by $H F_{*}(g)$. Note that $H F_{*}(g)$ is independent of the choice of an area form $\omega$ by Moser's theorem and naturality of Floer homology. By lemma 2.3 symplectomorphisms $\phi^{n}$ are also monotone for all $n>0$. Taking a dynamical point of view, we consider the iterates of monotone symplectomorphism $\phi$ and define the first symplectic zeta function of $\phi$ [14] as the following power series:

$$
\chi_{\phi}(z)=\exp \left(\sum_{n=1}^{\infty} \frac{\chi\left(H F_{*}\left(\phi^{n}\right)\right)}{n} z^{n}\right)
$$

where $\chi\left(H F_{*}\left(\phi^{n}\right)\right)$ is the Euler characteristic of Floer homology complex of $\phi^{n}$. Then $\chi_{\phi}(z)$ is an invariant of $g$, which we denote by $\chi_{g}(z)$. Let us consider the Lefschetz zeta function

$$
L_{\phi}(z):=\exp \left(\sum_{n=1}^{\infty} \frac{L\left(\phi^{n}\right)}{n} z^{n}\right)
$$


where $L\left(\phi^{n}\right):=\sum_{k=0}^{2}(-1)^{k} \operatorname{tr}\left[\phi_{* k}^{n}: H_{k}(M ; \mathbf{Q}) \rightarrow H_{k}(M ; \mathbf{Q})\right]$ is the Lefschetz number of $\phi^{n}$.

Theorem 3.1. 14] Symplectic zeta function $\chi_{\phi}(z)$ is a rational function of $z$ and

$$
\chi_{\phi}(z)=L_{\phi}(z)=\prod_{k=0}^{2} \operatorname{det}\left(I-\phi_{* k} \cdot z\right)^{(-1)^{k+1}} .
$$

Proof. If for every $n$ all the fixed points of $\phi^{n}$ are non-degenerate, i.e. for all $x \in \operatorname{Fix}\left(\phi^{n}\right), \operatorname{det}\left(\mathrm{id}-\mathrm{d} \phi^{n}(x)\right) \neq 0$, then we have( see section 2): $\chi\left(H F_{*}\left(\phi^{n}\right)\right)=$ $\sum_{x=\phi^{n}(x)} \operatorname{sign}\left(\operatorname{det}\left(\mathrm{id}-\mathrm{d} \phi^{n}(x)\right)\right)=L\left(\phi^{n}\right)$. If we have degenerate fixed points one needs to perturb equations (2.2) in order to define the Floer homology. The necessary analysis is given in [41] is essentially the same as in the slightly different situations considered in [6], where the above connection between the Euler characteristic and the Lefschetz number was firstly established.

In [14] we have defined the second symplectic zeta function for monotone symplectomorphism $\phi$ as the following power series:

$$
F_{\phi}(z)=\exp \left(\sum_{n=1}^{\infty} \frac{\operatorname{dim} H F_{*}\left(\phi^{n}\right)}{n} z^{n}\right) .
$$

Then $F_{\phi}(z)$ is an invariant of mapping class $g$, which we denote by $F_{g}(z)$.

Motivation for this definition was the theorem 2.4 and nice analytical properties of the Nielsen zeta function

$$
N_{\phi}(z)=\exp \left(\sum_{n=1}^{\infty} \frac{N\left(\phi^{n}\right)}{n} z^{n}\right)
$$

, see [10, 11, 40, 12]. We denote the numbers $\operatorname{dim} H F_{*}\left(\phi^{n}\right)$ by $N_{n}$. Let $\mu(d), d \in \mathbb{N}$, be the Möbius function.

Theorem 3.2. [14] Let $\phi$ be a non-trivial orientation preserving periodic diffeomorphism of least period $m$ of a compact connected surface $M$ of Euler characteristic $\chi(M)<0$. Then the zeta function $F_{\phi}(z)$ is a radical of a rational function and

$$
F_{\phi}(z)=\prod_{d \mid m} \sqrt[d]{\left(1-z^{d}\right)^{-P(d)}}
$$

where the product is taken over all divisors $d$ of the period $m$, and $P(d)$ is the integer $P(d)=\sum_{d_{1} \mid d} \mu\left(d_{1}\right) N_{d / d_{1}}$. 
Remark 3.1. Given a symplectomorphism $\phi$ of surface $M$, one can form the symplectic mapping torus $M_{\phi}^{4}=T_{\phi}^{3} \rtimes S^{1}$, where $T_{\phi}^{3}$ is usual mapping torus . Ionel and Parker [25] have computed the degree zero Gromov invariants [36] (these are built from the invariants of Ruan and Tian) of $M_{\phi}^{4}$ and of fiber sums of the $M_{\phi}^{4}$ with other symplectic manifolds. This is done by expressing the Gromov invariants in terms of the Lefschetz zeta function $L_{\phi}(z)$ [25]. The result is a large set of interesting non-Kahler symplectic manifolds with computational ways of distinguishing them. In dimension four this gives a symplectic construction of the exotic elliptic surfaces of Fintushel and Stern [16]. This construction arises from knots. Associated to each fibered knot $K$ in $S^{3}$ is a Riemann surface $M$ and a monodromy diffeomorphism $f_{K}$ of $M$. Taking $\phi=f_{K}$ gives symplectic 4-manifolds $M_{\phi}^{4}(K)$ with Gromov invariant $\operatorname{Gr}\left(M_{\phi}^{4}(K)\right)=A_{K}(t) /(1-t)^{2}=L_{\phi}(t)$, where $A_{K}(t)$ is the Alexander polynomial of knot $K$. Next, let $E^{4}(n)$ be the simply-connected minimal elliptic surface with fiber $F$ and canonicla divisor $k=(n-2) F$. Forming the fiber sum $E^{4}(n, K)=E^{4}(n) \#_{\left(F=T^{2}\right)} M_{\phi}^{4}(K)$ we obtain a symplectic manifold homeomorphic to $E^{4}(n)$. Then for $n \geq 2$ the Gromov and Seiberg-Witten invariants of $E^{4}(K)$ are $\operatorname{Gr}\left(E^{4}(n, K)\right)=S W\left(E^{4}(n, K)\right)=A_{K}(t)(1-t)^{n-2}$ [16, 25]. Thus fibered knots with distinct Alexander polynomials give rise to symplectic manifolds $E^{4}(n, K)$ which are homeomorphic but not diffeomorphic. In particular, there are infinitely many distinct symplectic 4-manifolds homeomorphic to $E^{4}(n)$ [16] .

In higher dimensions it gives many examples of manifolds which are diffeomorphic but not equivalent as symplectic manifolds. Theorem 3.1 implies that the Gromov invariants of $M_{\phi}^{4}$ are related to symplectic Floer homology of $\phi$ via zeta function $\chi_{\phi}(z)=L_{\phi}(z)$. We hope that the second symplectic zeta function $F_{\phi}(z)$ give rise to a new invariant of symplectic 4-manifolds [16].

3.2. Topological entropy and the Nielsen numbers. A basic relation between Nielsen numbers and topological entropy $h(f)$ [32] was found by N. Ivanov [27]. We present here a very short proof of Jiang of the Ivanov's inequality.

Lemma 3.3. [27]

$$
h(f) \geq \limsup _{n} \frac{1}{n} \cdot \log N\left(f^{n}\right)
$$

Proof. Let $\delta$ be such that every loop in $X$ of diameter $<2 \delta$ is contractible. Let $\epsilon>0$ be a smaller number such that $d(f(x), f(y))<\delta$ whenever $d(x, y)<2 \epsilon$. Let 
$E_{n} \subset X$ be a set consisting of one point from each essential fixed point class of $f^{n}$. Thus $\left|E_{n}\right|=N\left(f^{n}\right)$. By the definition of $h(f)$, it suffices to show that $E_{n}$ is $(n, \epsilon)$-separated. Suppose it is not so. Then there would be two points $x \neq y \in E_{n}$ such that $d\left(f^{i}(x), f^{i}(y)\right) \leq \epsilon$ for $o \leq i<n$ hence for all $i \geq 0$. Pick a path $c_{i}$ from $f^{i}(x)$ to $f^{i}(y)$ of diameter $<2 \epsilon$ for $o \leq i<n$ and let $c_{n}=c_{0}$. By the choice of $\delta$ and $\epsilon, f \circ c_{i} \simeq c_{i+1}$ for all $i$, so $f^{n} \circ c_{0} \simeq c_{n}=c_{0}$. such that This means $x, y$ in the same fixed point class of $f^{n}$, contradicting the construction of $E_{n}$.

This inequality is remarkable in that it does not require smoothness of the map and provides a common lower bound for the topological entropy of all maps in a homotopy class.

We recall Thurston classification theorem for homeomorphisms of surfase $M$ of genus $\geq 2$.

Theorem 3.4. [46] Every homeomorphism $\phi: M \rightarrow M$ is isotopic to a homeomorphism $f$ such that either

(1) $f$ is a periodic map; or

(2) $f$ is a pseudo-Anosov map, i.e. there is a number $\lambda>1$ (stretching factor) and a pair of transverse measured foliations $\left(F^{s}, \mu^{s}\right)$ and $\left(F^{u}, \mu^{u}\right)$ such that $f\left(F^{s}, \mu^{s}\right)=$ $\left(F^{s}, \frac{1}{\lambda} \mu^{s}\right)$ and $f\left(F^{u}, \mu^{u}\right)=\left(F^{u}, \lambda \mu^{u}\right)$; or

(3) $f$ is reducible map, i.e. there is a system of disjoint simple closed curves $\gamma=$ $\left\{\gamma_{1}, \ldots . ., \gamma_{k}\right\}$ in int $M$ such that $\gamma$ is invariant by $f\left(\right.$ but $\gamma_{i}$ may be permuted) and $\gamma$ has a $f$-invariant tubular neighborhood $U$ such that each component of $M \backslash U$ has negative Euler characteristic and on each(not necessarily connected) $f$-component of $M \backslash U, f$ satisfies (1) or (2).

The map $f$ above is called a Thurston canonical form of $\phi$. In (3) it can be chosen so that some iterate $f^{m}$ is a generalised Dehn twist on $U$. Such a $f$, as well as the $f$ in (1) or (2), will be called standard. A key observation is that if $f$ is standard, so are all iterates of $f$.

Lemma 3.5. 9] Let $f$ be a pseudo-Anosov homeomorphism with stretching factor $\lambda>1$ of surfase $M$ of genus $\geq 2$. Then

$$
h(f)=\log (\lambda)=\limsup _{n} \frac{1}{n} \cdot \log N\left(f^{n}\right)
$$


Lemma 3.6. [30] Suppose $f$ is a standard homeomorphism of surfase $M$ of genus $\geq 2$ and $\lambda$ is the largest stretching factor of the pseudo-Anosov pieces ( $\lambda=1$ if there is no pseudo-Anosov piece). Then

$$
h(f)=\log (\lambda)=\limsup _{n} \frac{1}{n} \cdot \log N\left(f^{n}\right)
$$

3.3. Asymptotic invariant. The growth rate of a sequence $a_{n}$ of complex numbers is defined by

$$
\operatorname{Growth}\left(a_{n}\right):=\max \left\{1, \limsup _{n \rightarrow \infty}\left|a_{n}\right|^{1 / n}\right\}
$$

which could be infinity. Note that $\operatorname{Growth}\left(a_{n}\right) \geq 1$ even if all $a_{n}=0$. When $\operatorname{Growth}\left(a_{n}\right)>1$, we say that the sequence $a_{n}$ grows exponentially.

Definition 3.2. We define the asymptotic invariant $F^{\infty}(g)$ of mapping class $g \in$ $\operatorname{Mod}_{M}=\pi_{0}\left(\operatorname{Diff} f^{+}(M)\right)$ to be the growth rate of the sequence $\left\{a_{n}=\operatorname{dim} H F_{*}\left(\phi^{n}\right)\right\}$ for a monotone representative $\phi \in \operatorname{Symp}^{m}(M, \omega)$ of $g$ :

$$
F^{\infty}(g):=\operatorname{Growth}\left(\operatorname{dim} H F_{*}\left(\phi^{n}\right)\right)
$$

Example 3.3. If $\phi$ is a non-trivial orientation preserving periodic diffeomorphism of a compact connected surface $M$ of Euler characteristic $\chi(M)<0$, then the periodicity of the sequence $\operatorname{dim} H F_{*}\left(\phi^{n}\right)$ implies that for the corresponding mapping class $g$ the asymptotic invariant

$$
F^{\infty}(g):=\operatorname{Growth}\left(\operatorname{dim} H F_{*}\left(\phi^{n}\right)\right)=1
$$

Example 3.4. Let $\phi$ be a monotone diffeomorphism of finite type of a compact connected surface $M$ of Euler characteristic $\chi(M)<0$ and $g$ a corresponding algebraically finite mapping class. Let $U$ be the open regular neighborhood of the $k$ reducing curves $\gamma_{1}, \ldots . ., \gamma_{k}$ in the Thurston theorem, and $M_{j}$ be the component of $M \backslash U$.Let $F$ be a fixed point class of $\phi$. Observe from [31] that if $F \subset M_{j}$, then ind $(F, \phi)=\operatorname{ind}\left(F, \phi_{j}\right)$. So if $F$ is counted in $N(\phi)$ but not counted in $\sum_{j} N\left(\phi_{j}\right)$ , it must intersect $U$. But we see from [31] that a component of $U$ can intersect at most 2 essential fixed point classes of $\phi$. Hence we have $N(\phi) \leq \sum_{j} N\left(\phi_{j}\right)$.For the monotone diffeomorphism of finite type $\phi$ maps $\phi_{j}$ are periodic. Applying last inequality to $\phi^{n}$ and using remark 2.9 we have

$$
0 \leq \operatorname{dim} H F_{*}\left(\phi^{n}\right)=\operatorname{dim} H_{*}\left(M_{\mathrm{id}}^{(n)}, \partial M_{\mathrm{id}}^{(n)} ; \mathbb{Z}_{2}\right)+N\left(\phi^{n} \mid\left(M \backslash M_{\mathrm{id}}^{(n)}\right)\right) \leq
$$




$$
\begin{gathered}
\leq \operatorname{dim} H_{*}\left(M_{\mathrm{id}}^{(n)}, \partial M_{\mathrm{id}}^{(n)} ; \mathbb{Z}_{2}\right)+N\left(\phi^{n}\right) \leq \\
\operatorname{dim} H_{*}\left(M_{\mathrm{id}}^{(n)}, \partial M_{\mathrm{id}}^{(n)} ; \mathbb{Z}_{2}\right)+\sum_{j} N\left(\left(\phi_{j}\right)^{n}\right)+2 k \leq \text { Const }
\end{gathered}
$$

by periodicity of $\phi_{j}$. Taking the growth rate in $n$, we get that asymptotic invariant $F^{\infty}(g)=1$.

Remark 3.5. For pseudo-Anosov mapping class $g \in \operatorname{Mod}_{M}=\pi_{0}\left(\operatorname{Diff}^{+}(M)\right)$ we have

$$
\operatorname{dim} H F_{*}(g)>N(g), F^{\infty}(g)>\limsup _{n \rightarrow \infty}\left|N\left(g^{n}\right)\right|^{1 / n}=h(\psi)=\lambda>1,
$$

where $N(g)$ denotes the Nielsen number of $g$ and $\psi$ is a standard(Thurston canonical form) representative of $g$.

Remark 3.6. For any mapping class $g \in \operatorname{Mod}_{M}=\pi_{0}\left(D i f f^{+}(M)\right)$ we have

$$
\operatorname{dim} H F_{*}(g) \geq N(g), F^{\infty}(g) \geq \lambda,
$$

where $\lambda$ is the largest stretching factor of pseudo-Anosov pieses of a standard( Thurston canonical form) representative of $g(\lambda:=1$ if there is no pseudo-Anosov piece).

\section{A generalisation of the Poincaré - Birkhoff theorem and Arnold CONJeCture. CONCLUding REMARKS}

A natural generalization of the Poincaré- Birkhoff theorem concerns the estimation of the number of fixed points of symplectomorphism $\phi \in \operatorname{Symp}\left(M^{2 n}, \omega\right)$. Actually this question was already raised by Birkhoff in [1]. He wrote with reference to the Poincaré - Birkhoff theorem: "Up to present time no proper generalisation of this theorem to higher dimensions has been found, so that its applications remains limited to dynamical systems with two degrees of freedom [1], page 150."

Based on the Poincaré - Birkhoff theorem Arnold formulated in the 1960s his famous conjecture: a Hamiltonian symplectomorphism( time-1 map of a time-dependent Hamiltonian flow) should have at least as many fixed points as a function on the manifold have critical points.

Let $\phi: M^{2 n} \rightarrow M^{2 n}$ be a Hamiltonian symplectomorphism of a compact symplectic manifold $\left(M^{2 n}, \omega\right)$. In the case when all fixed points of $\phi$ are nondegenerate the 
Arnold conjecture asserts that

$$
\# F i x(\phi) \geq \operatorname{dim} H_{*}(M, \mathbf{Q})=\sum_{k=0}^{2 n} b_{k}(M),
$$

where $2 n=\operatorname{dim} M, b_{k}(M)=\operatorname{dim} H_{k}(M, \mathbf{Q})$.

The Arnold conjecture in the nondegenerate case has now been proved in full generality. It was first proved by Eliashberg [8] for Riemann surfaces. For tori of arbitrary dimension it was proved in the celebrated paper by Conley and Zehnder[2]. The most important breakthrough was Floer's proof of the Arnold conjecture in the nondegenerate case for monotone symplectic manifolds [18]. His proof was based on Floer homology. His method has been pushed through by Fukaya-Ono[19], LiuTian [34] and Hofer-Salamon[22] to establish the nondegenerate case of the Arnold conjecture for all symplectic manifolds.

Some progress has been made with the original Arnold conjecture by Rudyak [43], using a development of Lusternik - Schnirelman theory called category weight. Using these ideas it was proved in [44] that the original conjecture holds in the case where degeneracies are allowed, provided that both classes [ $\omega$ ] and $c_{1}$ vanish on $\pi_{2}\left(M^{2 n}\right)$.

The Hamiltonian symplectomorphism $\phi$ is isotopic to identity map $i d_{M}$. In this case all fixed points $\phi$ are in the same Nielsen fixed point class. The Nielsen number of $\phi$ is 0 or 1 depending on Lefschetz number is 0 or not. So, the Nielsen number is very weak invariant to estimate the number of fixed points of $\phi$ for Hamiltonian symplectomorphism. From another side, as we saw in theorem 2.4, for the nontrivial periodic symplectomorphism $\phi$ of a surface, the Nielsen number of $\phi$ gives an exact estimation from below for the number of nondegenerate fixed points of $\phi$. These considerations lead us to the following question

Question 4.1. How to estimate the number of fixed points of symplectomorphism (not necessary Hamiltonian) $\phi \in \operatorname{Symp}\left(M^{2 n}, \omega\right)$ which has only nondegenerate fixed points?

This question can be considered as a generalisation of the Poincaré - Birkhoff theorem and as a generalisation of the Arnold conjecture in nondegenerate case .

4.1. Algebraically finite mapping class. If $\psi$ is a diffeomorphism of finite type of surface $M$ then $\psi \in \operatorname{Symp}^{m}(M, \omega)$ for some $\psi$-invariant form $\omega$. Suppose now that 
symplectomorphism $\phi$ has only non-degenerate fixed points and $\phi$ is Hamiltonian isotopic to $\psi$. Then $\phi \in \operatorname{Symp}^{m}(M, \omega)$ and $H F_{*}(\phi)$ is isomorphic to $H F_{*}(\psi)$. So, from theorem 2.9 it follows that

$$
\begin{gathered}
\# F i x(\phi) \geq \operatorname{dim} H F_{*}(\phi)=\operatorname{dim} H F_{*}(\psi)= \\
=\operatorname{dim} H_{*}\left(M_{\psi=\mathrm{id}}, \partial M_{\psi=\mathrm{id}} ; \mathbb{Z}_{2}\right)+N\left(\psi \mid\left(M \backslash M_{\psi=\mathrm{id}}\right)\right)= \\
=\sum_{k=0}^{2} b_{k}\left(M_{\psi=\mathrm{id}}, \partial M_{\psi=\mathrm{id}} ; \mathbb{Z}_{2}\right)+N\left(\psi \mid\left(M \backslash M_{\psi=\mathrm{id}}\right)\right)
\end{gathered}
$$

This estimation can be considered as a generalisation of the Poincaré - Birkhoff theorem and as a generalisation of Arnold conjecture for symplectomorphism with nondegenerate fixed points in algebraically finite mapping class, becouse it implies Arnold conjecture for $\psi=i d$. If $\psi$ is nontrivial orientation preserving periodic diffeomorphism then by theorem $2.4 \psi$ is a monotone symplectomorphism and by lemma 2.1 it has only nondegenerate fixed points. The theorem 2.4 implies an estimation

$$
\# F i x(\phi) \geq \operatorname{dim} H F_{*}(\phi)=\operatorname{dim} H F_{*}(\psi)=N(\psi)
$$

This estimation can be considered as a generalisation of Poincaré - Birkhoff theorem and Arnold conjecture for a nontrivial orientation-preserving periodic mapping class.

4.2. Pseudo-Anosov mapping class. For pseudo-Anosov "diffeomorphism" $\psi$ in given pseudo-Anosov mapping class $g$ we also have, as in theorems 2.4 2.8 and 2.9, a topological separation of fixed points [46, 31, 26], i.e the Nielsen number of pseudoAnosov "diffeomorphism" $\psi$ equals to the number of fixed points of $\psi$ and there are no connecting orbits between them. But we have the following difficulties. Firstly, a pseudo-Anosov "diffeomorphism" is a smooth and a symplectic automorphism only on the complement of the singular set of a pair of transverse measured foliations. Secondly, in the case of a pseudo-Anosov "diffeomorphism" we have to deal with fixed singular points of index $1-p$ where $p \geq 3$ is a number of prongs of fixed singularity . Such fixed points are degenerate from symplectic point of view and therefore need a local perturbation. The following theorem can be considered as a generalisation of Poincaré - Birkhoff theorem for a symplectomorphism with nondegenerate fixed points in a given pseudo-Anosov mapping class. 
Theorem 4.1. (Cotton-Clay [3], Fel'shtyn) If $\phi$ is a symplectomorphism with nondegenerate fixed points in given pseudo-Anosov mapping class $\{\phi\}=g=\{\psi\}$, then

$$
H F_{*}(\phi)=H F_{*}(g) \cong \mathbb{Z}_{2}^{\sum_{x \in \operatorname{Fix}(\psi)}|\operatorname{Ind}(x)|}
$$

and

$$
\# \text { Fix }(\phi) \geq \operatorname{dim} H F_{*}(\phi)=\operatorname{dim} H F_{*}(g)=\sum_{x \in \operatorname{Fix}(\psi)}|\operatorname{Ind}(x)|,
$$

where $\psi$ is a canonical pseudo-Anosov representative of $g$.

Proof. We describe main steps of the proof. Firstly we smooth the singular pseudoAnosov map $\psi$ locally near the singularities using Hamiltonian vector fields to get symplectomorphism $\hat{\psi}$ in the given mapping class $g$ with $|\operatorname{Ind}(x)|$ nondegenerate fixed points in the small neighborhood of $x$. The idea of such smoothing was suggested to the author by Kaoru Ono in 2005. Full details of this smoothing can be found in [3]. Then we show that all $|\operatorname{Ind}(x)|$ nondegenerate fixed points in the small neighborhood of $x$ are in the same Nielsen fixed point class. On the next step we prove that there are no pseudoholomorphic curves of index 1 between these nondegenerate fixed points. Then the boundary map in the Floer complex of $\hat{\psi}$ is zero and $\mathbb{Z}_{2}$-vector space $C F_{*}(\hat{\psi}):=\mathbb{Z}_{2}^{\# \operatorname{Fix}(\hat{\psi})}=\mathbb{Z}_{2}^{\sum_{x \in \operatorname{Fix}(\psi)}|\operatorname{Ind}(x)|}$. This immediately implies that $H F_{*}(\hat{\psi})$ is well defined and $H F_{*}(g)=H F_{*}(\hat{\psi}) \cong \mathbb{Z}_{2}^{\sum_{x \in \operatorname{Fix}(\psi)}|\operatorname{Ind}(x)|}$, and $\operatorname{dim} H F_{*}(\hat{\psi})=\sum_{x \in \operatorname{Fix}(\psi)}|\operatorname{Ind}(x)|$. On the last step of the proof, which was fully justified only recently by A. Cotton-Clay [3], we show that for any symplectomorphism $\phi$ in a pseudo-Anosov mapping class $g$ with nondegenerate fixed points $H F_{*}(\phi)$ is well defined and $H F_{*}(\phi)=H F_{*}(g)$.

Remark 4.2. A. Cotton-Clay found in [3] a nice combinatorial formula computing $H F_{*}(\hat{\psi})$ using train tracs .

Remark 4.3. In [7] Floer homology were calculated for certain class of pseudoAnosov maps which are compositions of positive and negative Dehn twists along loops in $M$ forming a tree-pattern. It is interesting to compare the results above with this calculation. 
4.3. Reducible mapping class. Recently, in the paper [3], A. Cotton-Clay calculated Seidel's symplectic Floer homology for reducible mapping classes. This result completing previous computations in the case of arbitrary compositions of Dehn twists along a disjoint collection of curves [41, in the case of periodic mapping classes [20, 14], as well as reducible mapping classes in which the map on each component is periodic [20] and in the case of certain compositions of Dehn twists, including some pseudo-Anosov maps [7].

In the case of reducible mapping classes a energy estimate forbids holomorphic discs from crossing reducing curves except when a pseudo-Anosov component meets an identity component ( with no twisting). Let us introduce some notation following [3. Recall the notation of $M_{i d}$ for the collection of fixed components as well as the tree types of boundary: 1) $\partial_{+} M_{i d}, \partial_{-} M_{i d}$ denote the collection of components of $\partial M_{i d}$ on which we've joined up with a positive(resp. negative) twist; 2) the collection of components of $\partial M_{\text {id }}$ which meet a pseudo-Anosov component will be denoted $\partial_{p} M_{\mathrm{id}}$. Additionally let $M_{1}$ be the collection of periodic components and let $M_{2}$ bethe collection of pseudo-Anosov components with punctures( i.e. before any perturbation) instead of boundary components wherever there is a boundary component that meets a fixed component. We further subdivide $M_{\mathrm{id}}$. Let $M_{a}$ be the collection of fixed components which don't meet any pseudo-Anosov components. Let $M_{b, p}$ be the collection of fixed components which meet one pseudo-Anosov component at a boundary with $p$ prongs. In this case, we assign the boundary components to $\partial_{+} M_{\text {id }}$ (this is an arbitrary choice). Let $M_{b, p}^{o}$ be the collection of the $M_{b, p}$ with each component punctured once. Let $M_{c, q}$ be the collection of fixed components which meets at least two pseudo-Anosov components such that the total number of prongs over all the boundaries is $q$. In this case, we assign at least one boundary component to $\partial_{+} M_{\text {id }}$ and at least one to $\partial_{-} M_{\text {id }}$ (and beyond that, it does not matter).

Theorem 4.2. ( A. Cotton-Clay [3]) Let $\phi$ be a perturbed standard form map [3] in a reducible mapping class $g$ with choices of the signs of components of $\partial_{p} M_{\mathrm{id}}$. Then $H F_{*}(\phi)$ is well-defined and

$$
\begin{gathered}
H F_{*}(g)=H F_{*}(\phi) \cong H_{*}\left(M_{a}, \partial_{+} M_{\mathrm{id}} ; \mathbb{Z}_{2}\right) \oplus \\
\oplus_{p}\left(H_{*}\left(M_{b}^{o}, \partial_{+} M_{b} ; \mathbb{Z}_{2}\right) \oplus\left(\mathbb{Z}_{2}\right)^{(p-1)\left|\pi_{0}\left(M_{b, p}\right)\right|}\right) \oplus
\end{gathered}
$$




$$
\begin{gathered}
\oplus_{q}\left(H_{*}\left(M_{c}, \partial_{+} M_{c} ; \mathbb{Z}_{2}\right) \oplus\left(\mathbb{Z}_{2}\right)^{q\left|\pi_{0}\left(M_{c, q}\right)\right|}\right) \oplus \\
\oplus \mathbb{Z}_{2}^{L\left(\phi \mid M_{1}\right)} \oplus C F_{*}\left(\phi \mid M_{2}\right),
\end{gathered}
$$

where $L\left(\phi \mid M_{1}\right)$ is the Lefschetz number of $\phi \mid M_{1}$, the $\mathbb{Z}_{2}^{L\left(\phi \mid M_{1}\right)}$ summand is all in even degree, the other two $\mathbb{Z}_{2}$ summands are all in odd degree, and $C F_{*}\left(\phi \mid M_{2}\right)$ denotes the chain complex for $\phi$ on the components $M_{2}$

Remark 4.4. The first summand and the $\mathbb{Z}_{2}^{L\left(\phi \mid M_{1}\right)}$ are as in R. Gautschi's Theorem 2.9 [20]. The last summand comes from the fact that there are no differentials in the Floer chain complex on the pseudo-Anosov components as in the proof of Theorem 4.1. The sums over $p$ and $q$ arise in the same manner as the first summand.

Corollary 4.3. As an application, A. Cotton-Clay gave recently [4, 5] a sharp lower bound on the number of fixed points of an area-preserving map( with nondegenerate fixed points) in any prescribed mapping class(rel boundary).

\subsection{Concluding remarks.}

- Due to P. Seidel [42] $\operatorname{dim} H F_{*}(\phi)$ is a new symplectic invariant of a fourdimensional symplectic manifold with nonzero first Betti number. This 4-manifold produced from symplectomorphism $\phi$ by a surgery construction which is a variation of earlier constructions due to McMullen-Taubes, Fintushel-Stern and J. Smith.We hope that our asymptotic invariant and symplectic zeta function $F_{\phi}(z)$ also give rise to a new invariants of contact 3- manifolds and symplectic 4-manifolds.

- Nielsen Floer homology. Let $\phi: M^{2 n} \rightarrow M^{2 n}$ be a symplectomorphism of a compact symplectic manifold $\left(M^{2 n}, \omega\right)$. Suppose that all fixed points of $\phi$ are nondegenerate.The symplectomorphism $\phi$ has finite number of Nielsen fixed point classes $F_{1}, F_{2}, \ldots, F_{n}$ and there is no pseudoholomorphic curves( instantons) between fixed points in the different Nielsen fixed point classes because there is no Nielsen discs between them. This simple observation strongly suggests to consider for every $i$ a local Floer complex $C F_{*}\left(\phi, F_{i}\right)$ generated by nondegenerate fixed points in the Nielsen fixed point class $F_{i}$ and to define for every $i$ a local Nielsen Floer homology $N F H_{*}\left(\phi, F_{i}\right)$ and then to define Nielsen Floer homology $N F H_{*}(\phi)$ of symplectomorphism $\phi$ as 
direct sum over all Nielsen fixed point classes of local Nielsen Floer homology:

$$
N F H_{*}(\phi)=\oplus_{F_{i}} N F H_{*}\left(\phi, F_{i}\right) .
$$

Question 4.5. Under which conditions on $\phi \in \operatorname{Symp}\left(M^{2 n}, \omega\right)$ Nielsen Floer homology are well-defined?

The results discussed in the present article strongly suggest that a calculation of Nielsen Floer homology and investigation of their properties should be important for a higher dimensional generalisation of Poincaré-Birkhoff theorem and Arnold conjecture in nondegenerate case. The author came to the idea of Nielsen Floer homology in 2001 after discussions with V. Turaev. A lack of a weak version of a monotonicity for the symplectomorphism $\phi$ prevented the development of this theory at that moment. Recently, Andrew Cotton-Clay [3] defined weak monotonicity for a given Nielsen fixed point class $F$ of symplectomorphism of surface and proved that Nielsen Floer homology are well defined in this case. The properties of $F$-weak monotone symplectomorphism of surface play a crucial role in his computation of Seidel's symplectic Floer homology for reducible mapping classes.

\section{REFERENCES}

1. G.D. Birkhoff, An extension of Poincaré's last geometric theorem, Acta mathematica, 47(1925), $297-311$.

2. C.Conley and E. Zehnder, The Birkhoff-Lewis fixed point theorem and a conjecture of V.I. Arnold. Inventiones Mathematicae, 73(1983), 33-49.

3. A. Cotton-Clay, Symplectic Floer homology of area-preserving surface diffeomorphisms, Preprint, Berkeley, June 2008.

4. A. Cotton-Clay, Symplectic Floer homology of pseudo-Anosov and reducible maps. A talk on the Northern California Symplectic Geometry Seminar, May 5, 2008.

5. A. Cotton-Clay, A sharp bound on fixed points of area-preserving surface diffeomorphisms, In preparation.

6. S. Dostoglou and D. Salamon, Self dual instantons and holomorphic curves, Annals of Math., 139 (1994), 581-640.

7. E. Eftekhary, Floer cohomology of certain pseudo-Anosov maps, J. Symplectic Geom.2(2004), no.3, 357-375.

8. Ya. Eliashberg, Estimates on the number of fixed points of area preserving transformations. Syktyvkar University Preprint, 1979. 
9. A. Fathi, F. Laudenbach, and V. Poénaru, Travaux de Thurston sur les surfaces, Astérisque, vol. 66-67, Soc. Math. France, 1979.

10. A. L. Fel'shtyn, New zeta function in dynamic. in Tenth Internat. Conf. on Nonlinear Oscillations, Varna, Abstracts of Papers, Bulgar. Acad. Sci., 1984, 208

11. A.L. Fel'shtyn, New zeta functions for dynamical systems and Nielsen fixed point theory. in : Lecture Notes in Math. 1346, Springer, 1988, 33-55.

12. A. Fel'shtyn, Dynamical zeta functions, Nielsen theory and Reidemeister torsion, Mem. Amer. Math. Soc. 147 (2000), no. 699, xii+146. MR2001a:37031

13. A. L. Fel'shtyn and R. Hill, The Reidemeister zeta function with applications to Nielsen theory and a connection with Reidemeister torsion, K-theory 8 no.4 (1994), 367-393.

14. Fel'shtyn A.L. Floer homology, Nielsen theory and symplectic zeta functions. Proceedings of the Steklov Institute of Mathematics, Moscow, vol. 246, 2004, pp. 270-282.

15. Fel'shtyn A.L. New directions in Nielsen-Reidemeister theory. E-print arxiv: math.GR/0712.2601.

16. R. Fintushel, R. Stern, Knots, links and 4-manifolds, Invention. Math. 134(1998), 363-400.

17. A. Floer. Morse theory for Lagrangian intersections. J. Differential Geom., 28(1988), 513-547.

18. A. Floer. Symplectic fixed points and holomorphic spheres. Comm. Math. Phys., 120(2),575$611,1989$.

19. K. Fukaya, K. Ono, Arnold conjecture and Gromov-Witten invariants for general symplectic manifolds, Topology 38(1999), 933-1048.

20. R. Gautschi. Floer homology of algebraically finite mapping classes J. Symplectic Geom. 1(2003), no.4, 715-765.

21. M. Gromov. Pseudoholomorphic curves in symplectic manifolds. Invent. Math.82(1985), 307347.

22. H. Hofer and D. Salamon, Floer homology and Novikov's ring, in Floer Memorial Volume, 1995, 483-524, Birkhauser.

23. N. V. Ivanov, Automorphisms of Teichm uller modular groups, Lecture Notes in Mathematics 1346 Springer, Berlin, 1988.

24. M. Hutchings, M. Sullivan, The periodic Floer homology of Dehn twist, Alg. Geom. Topol. $5(2005), 301-354$.

25. E.-N. Ionel, Th. Parker, Gromov invariants and symplectic maps, Math. Ann. 314(1999), 127158.

26. N. V. Ivanov, Nielsen numbers of maps of surfaces. Journal Sov. Math., 26, (1984).

27. N. V. Ivanov, Entropy and the Nielsen Numbers. Dokl. Akad. Nauk SSSR 265 (2) (1982), 284-287 (in Russian); English transl.: Soviet Math. Dokl. 26 (1982), 63-66.

28. B. Jiang, Lectures on Nielsen fixed point theory, Contemp. Math., vol. 14, Amer. Math. Soc., Providence, RI, 1983. 
29. B. Jiang. Fixed point classes from a differentiable viewpoint. In Fixed point theory, Lecture Notes in Math.,vol. 886, 163-170. Springer, 1981.

30. B. Jiang, Estimation of the number of periodic orbits. Pacific Jour. Math., 172(1996), 151-185.

31. B. Jiang and J. Guo. Fixed points of surface diffeomorphisms. Pac. J. Math., 160(1):67-89, 1993.

32. A. Katok, B. Hasselblatt, Introduction to the modern theory of dynamical systems, Cambridge University Press, 1995.

33. P. Kronheimer, T. Mrowka, Monopoles and Three - Manifolds, Cambridge University Press, 2007.

34. G. Liu and G. Tian, Floer homology and Arnold conjecture, J.Diff.Geom., 49(1998), 1-74.

35. D. McDuff and D. A. Salamon. Introduction to symplectic topology. Oxford Mathematical Monographs. Oxford Science Publications, 1998.

36. D. McDuff and D. A. Salamon. J-holomorphic Curves and Symplectic Topology. AMS Colloquium Publications, Vol. 52, 2004.

37. J. Moser. On the volume elements on a manifold. Trans. Amer. Math. Soc., 120:286-294, 1965.

38. P. Ozsváth, Z. Szabó, Holomorphic disks and topological invariants for closed 3-manifolds, Annals of Math. 159(2004), 1027-1158.

39. J. Nielsen. Surface transformations of algebraically finite type. Danske Vid. Salsk. Math.-Phys., $21,1944$.

40. V. B. Pilyugina and A. L. Fel'shtyn, The Nielsen zeta function. Funktsional. Anal. i Prilozhen. 19 (4) (1985), 61-67 (in Russian); English transl.: Functional Anal. Appl. 19 (1985), 300-305.

41. P. Seidel. Symplectic Floer homology and the mapping class group. Pacific J. Math. 206(2002), no. 1, 219-229.

42. P. Seidel. Braids and symplectic four-manifolds with abelian fundamental group. Turkish J. Math. 26(2002), no.1, 93-100.

43. Yu. Rudyak, On category weight and its applications, Topology 38(1999), no.1, 37-55.

44. Yu. Rudyak, J. Oprea, On the Lusternik - Schnirelmann category of symplectic manifolds and the Arnold conjecture,Math.Z.230(1999), no.4, 673-678.

45. W. Thurston, The geometry and topology of 3-manifolds. Princeton University, 1978.

46. W. P. Thurston. On the geometry and dynamics of diffeomorphisms of surfaces. Bull. Amer. Math. Soc., 19(2):417-431, 1988.

Institute of Mathematics, University of Szczecin, Ul. Wielkopolska 15, 70-451

Szczecin, Poland and Department of Mathematics, Boise State University, 1910

University Drive, Boise, IDAho, 83725-155, USA

E-mail address: felshtyn@diamond.boisestate.edu, felshtyn@mpim-bonn.mpg.de 